\title{
Revisiting parent-child interactions in early childhood as relevant factor in the development of ADHD
}

\author{
P. J. Havinga ${ }^{1}$ - C. A. Hartman ${ }^{2}$ J. C. Visser ${ }^{3} \cdot$ N. Rommelse ${ }^{1,3}$
}

Published online: 11 September 2019

○) Springer-Verlag GmbH Germany, part of Springer Nature 2019

In this issue of European Child and Adolescent Psychiatry, Joseph and colleagues investigated the presentation of depressive symptoms in a large, community-based sample of young children (6-8 years) with and without ADHD. This study extends previous work by showing that increased depressive symptom levels are already present in children with ADHD as young as age six. Sadness, irritability, insomnia, psychomotor agitation, feeling bad about oneself, difficulty concentrating, and making decisions were among the symptoms more frequently occurring in children with ADHD as compared to those without. These findings highlight the importance of early detection and treatment of depressive symptoms in young children with ADHD.

Although mechanisms explaining the high frequency of depressive symptoms in these young children were not investigated, the authors notice that caregiver's mental health difficulties may be a relevant factor. This would indeed be in line with the existing literature, showing that (1) parents of children with ADHD often experience mental health problems themselves (including ADHD and depression) $[1,2](2)$ depression in parents increases the development of depressive symptoms in (very) young children amongst

\footnotetext{
N. Rommelse

Nanda.Lambregts-Rommelse@ radboudumc.nl

P. J. Havinga

petra.havinga@radboudumc.nl

C. A. Hartman

c.hartman@accare.nl

J. C. Visser

janne.visser@radboudumc.nl

1 Department of Psychiatry, Radboudumc, Nijmegen, The Netherlands

2 Interdisciplinary Center Psychopathology and Emotion Regulation (ICPE), University Medical Center Groningen, University of Groningen, Groningen, The Netherlands

3 Karakter Child and Adolescent Psychiatry University Center, Nijmegen, The Netherlands
}

other factors through influencing parenting practices [3, 4], and (3) treatment of parental depression and/or parent-child interaction therapy may reduce depression in young children [5]. Indirectly these findings support the relevance of including the early parent-child interaction as relevant domain in the search for early risk pathways towards the development of ADHD and its comorbidities.

We propose that Emotion Dysregulation (ED) in parent-child interaction is such a risk pathway. Parents play a pivotal role in supporting and promoting emotion regulation during early childhood. The literature on children's social development, a field of research which has developed virtually independent of the ADHD literature, clearly shows that parents are the most influential in facilitating and promoting young children's ability to develop effective strategies to regulate their emotions. The early development of effective emotion regulation abilities-especially in easily dysregulated children - is strongly dependent on a context of safe, supportive, and well-adapted parent-child relationships, wherein parents are able to support and stimulate the child's emotion regulation capacities [6]. Of great relevance is that a recent study indicates that parental use of emotion socialization practices (both supportive and non-supportive) has a relatively stronger impact on children with high ADHD symptomatology [7]. This suggests that parental use of adaptive emotion socialization practices may be particularly relevant in the context of susceptibility towards developing ADHD.

Reversely, children do also play a pivotal role in influencing parental emotion regulation. Studies show that parents who have toddlers with disruptive behavior problems exhibited more hostile attributional biases and emotion flooding, as well as more negative discipline practices [8]. Toddlers who continue to exhibit strong emotion dysregulation in the context of challenging temperamental styles (so-called 'difficult temperament') tend to elicit more negative, unresponsive, and/or punitive reactions from parents during this age period [6]. Children's behavior problems and parents' 
unsupportive emotion socialization practices can become mutually maintaining and reinforcing coercive patterns in a negative family environment, with a poor match between child and parent characteristics (i.e., poor 'goodness of fit') [9]. These patterns cannot completely be accounted for by shared genetic influences between child and parents, since in adoption families, the same aversive parent-child interaction spirals have been documented [10].

Importantly, a review showed that EDs seem to be a key precursor of ADHD that manifests itself already in infants at a very young age in a substantial subset of children who later develop ADHD [11]. A recently published study confirms this finding showing that excitability (emotion dysregulation of positive emotions) in young children age 3-5 years predicts an ADHD diagnosis at age 14-19 years [12]. Moreover, already in infancy, excessive infant crying during the 13th week after birth doubles the risk of hyperactivity at age 5-6 [13]. Also ED during middle/late childhood is predictive for experiencing later ADHD symptoms [14]. It may well be the case that the participants described in the study of Joseph and colleagues may have shown depressive symptoms at an even earlier age than now investigated, an age preceding a clinical diagnosis of ADHD. As ADHD runs in families, parent-child reciprocal influences on emotion dysregulation are likely to be particularly prevalent in families with a high familial risk for ADHD: (1) ED is highly prevalent in adults with ADHD; (2) about half of the parents with ADHD have a child who will develop ADHD and about half of these children will have ED levels that are not observed in the general population; and (3) children exhibiting ED show increased sensitivity to parental ED as compared to children without ED being exposed to the same parenting practices. Goodness of fit between parent and child characteristics is, therefore, a critical factor in the context of ADHD risk [9], and is at the same time harder to accomplish given that the ED problems run in these families. A recent study shows that already during pregnancy, poor maternal emotion regulation is predictive of ED in newborns [15]. Persistence thereof may pave the way to increasingly poor self-control (i.e., the capacity to override automatic responses through controlled processing) relevant when entering primary school. This poor self-control surfaces in the context of school-challenges as a poor ability to maintain focus, sit still, and await turn, which are important symptoms of ADHD. Therefore, intervention improving parent-child emotion regulation in high ADHD-risk parent-child relationships in early childhood may in theory have a positive impact on the onset of ADHD in the child.

Several well-described early intervention programs have been developed and tested for preschoolers (4-7 year olds) displaying high levels of ADHD and/or oppositional behavior, such as the Triple-P, New Forest Parenting Programme, and Incredible Years. These programs have been shown to have moderate effects on reducing ADHD symptoms and moderate-to-large effects on reducing behavioral problems. To our knowledge, only two small-scale studies have examined programs specifically targeting parental emotion socialization in the context of ADHD [16, 17]. These studies show the potential of improving child's ED and ADHD/externalizing problems through intervention directed at improving parental emotion socialization practices. Importantly, these studies selected children that already showed clear manifestations of ADHD, instead of selecting younger children with elevated ED, but not yet full ADHD. It thus remains untested whether the liability for ADHD will not unfold into the full extreme by improving the parent-child interactions supporting and promoting emotion regulation abilities in young children.

As we reviewed above, early intervention in young children with ADHD has gained some ground in the past decade, but research on prevention is virtually absent. Thorough studies focusing on the pre-onset period are scarce, especially in early years (from birth to preschool age), while such studies are critical in untangling the causes from the consequences of ADHD. The main reason for this is the current dominant deterministic view on the causes of ADHD, postulating that onset of this condition is not amenable to change. Indeed, ADHD is known to be among the most heritable psychiatric disorders with genetic factors accounting for $\sim 70-75 \%$ of the variance. However, highly heritable conditions can also be highly susceptible to environmental conditions, with the prototypic example of significant increases in height due to improved nutrition in the last 100 years. Interventions cannot change the genetic liability of ADHD as it runs through families affected by ADHD, but can improve the circumstances for young offspring, such that their liability may not unfold into the full extreme. Further work is required to increase insights in the developmental processes leading to an onset of ADHD and to study whether early intervention targeted at these processes can prevent the development of clinical ADHD. Such studies may ultimately pave the way for early screening and intervention programs for high-risk children.

\section{References}

1. Chronis AM, Lahey BB, Pelham WE Jr, Kipp HL, Baumann BL, Lee SS (2003) Psychopathology and substance abuse in parents of young children with attention-deficit/hyperactivity disorder. $\mathbf{J}$ Am Acad Child Adolesc Psychiatry 42(12):1424-1432

2. Wesseldijk LW, Dieleman GC, van Steensel FJA et al (2018) Risk factors for parental psychopathology: a study in families with children or adolescents with psychopathology. Eur Child Adolesc Psychiatry $27(12): 1575-1584$

3. Ahun MN, Consoli A, Pingault JB et al (2018) Maternal depression symptoms and internalising problems in the offspring: the 
role of maternal and family factors. Eur Child Adolesc Psychiatry 27(7):921-932

4. Goodman SH (2007) Depression in mothers. Annu Rev Clin Psychol 3:107-135

5. Cuijpers P, Weitz E, Karyotaki E, Garber J, Andersson G (2015) The effects of psychological treatment of maternal depression on children and parental functioning: a meta-analysis. Eur Child Adolesc Psychiatry 24(2):237-245

6. Steinberg EA, Drabick DA (2015) A developmental psychopathology perspective on ADHD and comorbid conditions: the role of emotion regulation. Child Psychiatry Hum Dev 46(6):951-966

7. Breaux RP, McQuade JD, Harvey EA, Zakarian RJ (2018) Longitudinal associations of parental emotion socialization and children's emotion regulation: the moderating role of ADHD symptomatology. J Abnorm Child Psychol 46(4):671-683

8. Mence M, Hawes DJ, Wedgwood L et al (2014) Emotional flooding and hostile discipline in the families of toddlers with disruptive behavior problems. J Fam Psychol 28(1):12-21

9. Newland RP, Crnic KA (2017) Developmental risk and goodness of fit in the mother-child relationship: links to parenting stress and children's behaviour problems. Infant Child Dev 26(2):e1980. https://doi.org/10.1002/icd.1980

10. Harold GT, Leve LD, Barrett D et al (2013) Biological and rearing mother influences on child ADHD symptoms: revisiting the developmental interface between nature and nurture. J Child Psychol Psychiatry 54(10):1038-1046

11. Visser JC, Rommelse NN, Greven CU, Buitelaar JK (2016) Autism spectrum disorder and attention-deficit/hyperactivity disorder in early childhood: a review of unique and shared characteristics and developmental antecedents. Neurosci Biobehav Rev 65:229-263

12. Vogel AC, Jackson JJ, Barch DM, Tillman R, Luby JL (2019) Excitability and irritability in preschoolers predicts later psychopathology: the importance of positive and negative emotion dysregulation. Dev Psychopathol 31:1067-1083

13. Smarius LJ, Strieder TG, Loomans EM et al (2017) Excessive infant crying doubles the risk of mood and behavioral problems at age 5: evidence for mediation by maternal characteristics. Eur Child Adolesc Psychiatry 26(3):293-302

14. Wang B, Brueni LG, Isensee C et al (2018) Predictive value of dysregulation profile trajectories in childhood for symptoms of ADHD, anxiety and depression in late adolescence. Eur Child Adolesc Psychiatry 27(6):767-774

15. Ostlund BD, Vlisides-Henry RD, Crowell SE et al (2019) Intergenerational transmission of emotion dysregulation: part II. Developmental origins of newborn neurobehavior. Dev Psychopathol 31(3):833-846

16. Herbert SD, Harvey EA, Roberts JL, Wichowski K, Lugo-Candelas CI (2013) A randomized controlled trial of a parent training and emotion socialization program for families of hyperactive preschool-aged children. Behav Ther 44(2):302-316

17. Chronis-Tuscano A, Lewis-Morrarty E, Woods KE, O'Brien KA, Mazursky-Horowitz H, Thomas SR (2016) Parent-child interaction therapy with emotion coaching for preschoolers with attention-deficit/hyperactivity disorder. Cogn Behav Pract 23(1):62-78. https://doi.org/10.1016/j.cbpra.2014.11.001 